\title{
Chirurgische Zugänge zum Tibiakopf
}

Tim-Nicolas Hissnauer, Matthias Krause, Jannik Frings, Karl-Heinz Frosch

\section{Einleitung}

Wenn auch mit einer Inzidenz von 10,3/100000 relativ selten, sind Tibiakopffrakturen häufig komplex und können auch für den erfahrenen Operateur eine große chirurgische Herausforderung darstellen [1]. Um posttraumatische Deformitäten und fehlverheilte Frakturen zu vermeiden, ist die Wahl des geeigneten chirurgischen Zugangs zum Tibiakopf entscheidend und Voraussetzung für ein gutes postoperatives Ergebnis. Ziel der operativen Versorgung am Tibiakopf muss die bestmögliche anatomische Rekonstruktion der Gelenkfläche sein, um klinisch relevante Pseudoinstabilitäten sowie Achsdeformitäten der Beinachse zu vermeiden und die Rate posttraumatischer Kniegelenksarthrosen zu minimieren [2-4]. Insbesondere bei den mit 34,5\% besonders häufigen bikondylären Frakturen ist die in der Literatur beschriebene Rate für posttraumatische Arthrosen mit $44 \%$ sehr hoch $[5,6]$. Sofern aufgrund potenziell bestehender Begleitverletzungen möglich, sollte postoperativ eine langfristige Immobilisation vermieden und dem Patienten eine frühfunktionelle Nachbehandlung ermöglicht werden [7].

\section{Merke}

Bei Tibiakopffrakturen handelt es sich mehrheitlich um komplexe und chirurgisch anspruchsvolle Gelenkfrakturen, die bei inadäquater Versorgung zu klinisch relevanten Achsdeformitäten, Pseudoinstabilitäten und zu posttraumatischen Kniegelenksarthrosen führen können.

\section{Klassifikation und Indikation}

\section{Klassifikation}

Da die Morphologie der Fraktur den geeigneten chirurgischen Zugang vorgibt, ist es wichtig, Tibiakopffrakturen exakt und behandlungsorientiert zu klassifizieren. Hierfür ist die präoperative computertomografische Bildgebung obligat. Die bekanntesten Klassifikationen zur Einteilung von Tibiakopffrakturen stellen die AO-Klassifikation und die Klassifikation nach Schatzker sowie nach Moore dar. Diese Klassifikationen betrachten 2-dimensional die anteroposteriore Frakturmorphologie (AO und Schatzker) und gleichzeitige Weichteilverletzungen bei Luxationsfrakturen (Moore). Sie sind zwar gebräuchlich, besitzen allerdings nur eine mäßige Interobserver-Reliabilität [8-10].
Aufgrund ihres vorrangig deskriptiven Charakters und nur mangelnder Erfassung der Frakturlokalisation, reichen die genannten Klassifikationen in vielen Fällen nicht aus, um den chirurgischen Zugang angemessen zu planen. Daher haben sich zuletzt neuere, computertomografiebasierte Klassifikationen etabliert, welche die Fraktur in ihrer Dreidimensionalität erfassen und somit behandlungsorientierter sind. Das biomechanische Dreisäulenmodell nach Luo et al. teilt den Tibiakopf in eine anterolaterale, anteromediale und posteriore Säule und bietet eine Fraktureinteilung, anhand derer sich der chirurgische Zugang zum Tibiakopf alternativ planen lässt [11, 12]. Exakt kann die Frakturlokalisation durch die „Zehensegmentklassifikation“ erfasst werden [13]. Durch die Aufteilung des Tibiakopfes in 4 Quadranten und 10 Segmente ermöglicht diese Klassifikation eine differenzierte präoperative Planung, sodass Stufen oder Spalten auf Quadranten- und Segmentebene durch den passenden chirurgischen Zugang oder deren Kombination gezielt adressiert werden können [13] ( $\bullet$ Abb. 1).

\footnotetext{
Merke

Die Morphologie der Fraktur gibt den idealen chirurgischen Zugang vor.

Die dreidimensionale Analyse der Tibiakopffraktur mithilfe neuer, CT-basierter Klassifikationssysteme kann präoperativ bei der Wahl des geeigneten Zugangs behilflich sein!
}

\section{Indikationen zur operativen Versorgung}

Zur Indikationsprüfung der operativen Versorgung einer Tibiakopffraktur sollten folgende Fragen gestellt werden $[14,15]$ :

\footnotetext{
Merke

- 1. Wie groß ist die intraartikuläre Stufen- bzw. Spaltbildung in den lasttragenden Gelenkabschnitten?

- 2. In welchem Ausmaß ist die Gelenkfläche abgesunken oder abgekippt?

- 3. Wie groß ist die konsekutive Verbreiterung des Tibiakopfes?

- 4. Liegt eine koronare oder sagittale Achsabweichung vor?

- 5. Ist das mediale Tibiaplateau mitbetroffen?

- 6. Welche Begleitverletzungen liegen vor:

- assoziierte Diaphysenfraktur oder ipsilaterale Fibulafraktur?
} 


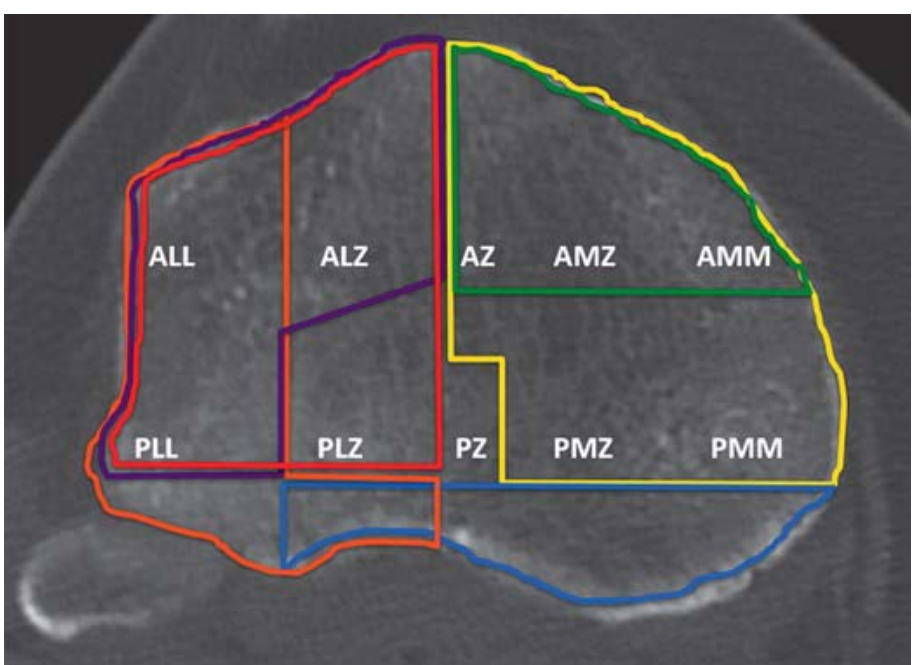

- Abb. 1 Schematische Darstellung der Zehnsegmentklassifikation nach Frosch sowie der zugangsbezogenen Erreichbarkeit. Grün Anteromedialer Zugang [14]; blau posteriorer posteromedialer Zugang [11, 15, 16]; bordeaux anterolateraler Zugang [14]; orange modifizierter posterolateraler Zugang [16]; rot erweiterter lateraler Zugang [17]; gelb erweiterter medialer Zugang [18] (ALL: anterolaterolateral, ALZ: anterolaterozentral, AZ: anterozentral, AMZ: anteromediozentral, AMM: anteromediomedial, PLL: posterolaterolateral, PLZ: posterolaterozentral, PZ: posterozentral, PMZ: posteromediozentral, PMM: posteromediomedial).

- instabile ligamentäre Verletzungen?

- Meniskusläsionen und Knorpelschäden

- 7. Droht ein Kompartmentsyndrom?

- 8. Handelt es sich um eine offene Fraktur?

Eine Operationsindikation ist gegeben, wenn ... [14, 15]:

\section{Merke}

- 1. ... die intraartikuläre Stufe $>2$ mm beträgt.

- 2. ... die Gelenkfläche $>5^{\circ}$ abgesunken ist.

- 3. ... die Verbreiterung des Tibiakopfes $>5 \mathrm{~mm}$ beträgt.

- 4. ... die Achsabweichung $>5^{\circ}$ misst.

- 5. ...ja (es besteht keine zwingende Indikation bei fissuralen oder nicht dislozierten Frakturen).

- 6. -8. ...ja.

Bei der Planung der Operation sollte neben dem Allgemeinzustand des Patienten und weiteren Begleitverletzungen auch der Weichteilschaden am frakturierten Tibiakopf berücksichtigt werden. Bei stark geschwollenen Weichteilen sollte die definitive Versorgung verzögert und erst nach adäquatem Abschwellen idealerweise nach 5-8 Tagen stattfinden. Bei offenen oder instabilen Verletzungen sollte zunächst die unmittelbare, notfallmäßige Ruhigstellung in einem kniegelenkübergreifenden Fixateur externe erfolgen [16]. Für den Fall eines manifesten oder drohenden Kompartmentsyndroms muss der Zeitpunkt der definitiven osteosynthetischen Versorgung ab- gewogen werden. Eine leitlinienbasierte Empfehlung findet sich diesbezüglich nicht. Analysen größerer Kollektive deuten daraufhin, dass zur Minimierung des Infektionsrisikos der sekundäre Wundverschluss nach stattgehabter Kompartmentspaltung vor der definitiven Versorgung stattfinden sollte [17-19].

\section{Chirurgische Zugänge}

Es existiert eine Vielzahl chirurgischer Zugänge zum Tibiakopf. Diese Darstellung soll sich auf die Operationszugänge fokussieren, welche aus Sicht der Autoren von Bedeutung sind und im klinischen Alltag relevante Anwendung finden. $>$ Abb. 1 stellt die segmentabhängige Adressierbarkeit durch den jeweiligen chirurgischen Zugang zum Tibiakopf dar. Hier sollte berücksichtigt werden, dass insbesondere posterolaterale Frakturfragmente nicht ausreichend durch anterolateral eingebrachtes Osteosynthesematerial gefasst werden können [20,21]. Daher sollten Fragmente im posterolaterozentralen Segment und posteromedialen Quadranten auch bevorzugt direkt über dorsale Zugänge versorgt werden [22-24]. - Abb. 2 zeigt die mögliche Positionierung der Plattenlage in Abhängigkeit von der Lage des Patienten.

\section{Anterolateraler Zugang}

Da bei 88,8\% der Tibiakopffrakturen das laterale Tibiaplateau isoliert oder in Kombination mitbetroffen ist [5], findet der anterolaterale Standardzugang zum Tibiakopf häufig Anwendung. Der Patient wird in Rückenlage gelagert. Empfehlenswert ist eine gerade Schnittführung vom Epicondylus femoris lateralis und ein wenig hinter dem Tuberculum Gerdy nach distal ziehend. Zudem sollte abhängig von der Frakturmorphologie berücksichtigt werden, ob der Hautschnitt bevorzugt anterolateral, lateral oder posterolateral durchgeführt wird. Ersterer ermöglicht eher die Adressierung eines Tuberositas-Fragmentes. Im Rahmen der weiteren Präparation in die Tiefe sollte vermieden werden, dass ein iatrogenes subkutanes Weichteil-Décollement entsteht. Zunächst erfolgt die Darstellung der Faszie des M. tibialis anterior, des Tractus iliotibialis sowie der Tibiavorderkante. Nach anschließender längsverlaufender Inzision des Tractus iliotibialis bis über das Tuberculum Gerdy an der Tibiavorderkante entlang kann der Tractus nach ventral vom Tuberculum Gerdii gelöst werden. Das Areal von der Tuberositas tibiae bis nach dorsal zum Vorderrand des Fibulaköpfchens kann exponiert werden. Es erfolgt ein submeniskaler Zugang zum Gelenk, das Lig. meniscotibiale wird hierzu durchtrennt, $2 \mathrm{~mm}$ sollten tibial für eine Refixation stehen bleiben. So gelingt Einsicht auf das anterolaterale Drittel des lateralen Tibiaplateaus sowie über den gesamten lateralen Rand des Plateaus. Empfehlenswert ist im Rahmen der Eröffnung der Faszie des M. tibialis anterior eine Faszienbrücke von ca. $5 \mathrm{~mm}$ zu belassen, sodass eine gute Weichteildeckung des Osteosynthesematerials gelingt. Posterolateral lokalisierte Frakturen lassen sich über den 

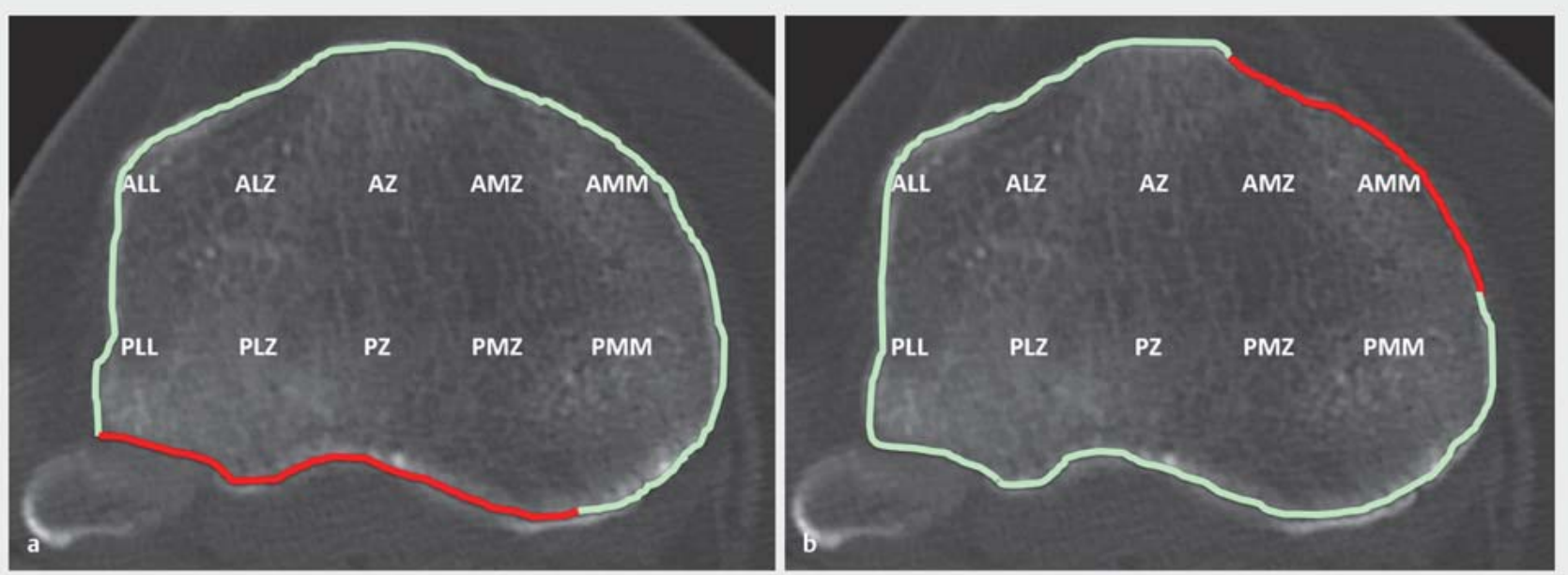

- Abb. 2 Mögliche Positionierung einer Platte zur osteosynthetischen Versorgung in Rückenlage (a) und Seit-/Bauchlage (b). Grün: Eine operative Adressierung über eine in diesem Bereich positionierte Platte zur Abstützung ist möglich; rot: eine Abstützung über eine in diesem Bereich positionierte Platte ist nicht möglich.

anterolateralen Zugang weder adäquat einsehen noch erfolgreich reponieren und osteosynthetisch adressieren. Insbesondere Frakturen mit Lokalisation im posterolateralzentralen Segment lassen sich nicht suffizient über den anterolateralen Zugang darstellen ( $\bullet$ Abb. 1)

Merke

Der anterolaterale Standardzugang ermöglicht lediglich das Einsehen des anterioren Quadranten sowie in das posterolaterallaterale Segment der Gelenkfläche! Das posterolateralzentrale Segment kann damit nicht visualisiert werden!

\section{Posterolateraler Zugang}

In der Literatur finden sich zahlreiche Beschreibungen von posterolateralen Zugangswegen zum Tibiakopf. Hierbei werden direkt posteriore, posterolaterale mit und ohne Osteotomie des Fibulakopfes, posteromediale oder modifiziert anterolaterale Zugänge beschrieben [25]. Die Autoren halten den posterolateralen Zugang ohne Fibulaosteotomie [22] für den geeignetsten Zugang, da weichteilschonend eine sehr gute Übersicht über die dorsalen und lateralen Segmente des Tibiakopfes erlangt werden kann. Die Lagerung des Patienten erfolgt bevorzugt in Seitenlage, aber auch in Bauchlage ist dieser Zugang durchführbar. Bei Positionierung des Patienten auf der Seite sollte das Kniegelenk auf einer gewickelten Rolle aufliegen, sodass das Gewicht des Beines selbst einen Varusstress verursacht, welcher den lateralen Gelenkspalt aufdehnt und zu einer besseren Einsicht verhilft. Des Weiteren kann hierbei durch die Ligamentotaxis in vielen Fällen bereits eine partielle Reposition der lateralen und posterolateralen Frakturfragmente erreicht werden. Der Hautschnitt erfolgt über eine Länge von ca. 10-12 cm entlang des $\mathrm{M}$. biceps femoris direkt über dem Fibulakopf beginnend etwa $4 \mathrm{~cm}$ oberhalb des Gelenkspaltes, knapp proximal des lateralen Epicondylus. Der Fibulakopf dient dabei als anatomischer Orientierungspunkt. Über den posterolateralen Zugang können ventral und dorsal des Fibulakopfes 2 Fenster angelegt werden $(\bullet$ Abb. 3).

Dorsal des Fibulakopfes erfolgt die Präparation nach Durchtrennung der Haut und des subkutanen Fettgewebes an der dorsalseitigen Begrenzung des M. biceps femoris. Direkt nach Eröffnen der Faszie kann der N. peronaeus an der Hinterkante des M. biceps femoris identifiziert werden. Der Nerv sollte sorgfältig präpariert werden und nur so weit mobilisiert werden, dass er während der Operation stets identifiziert werden kann. Anschließend erfolgt die stumpfe digitoklastische Präparation zwischen dem lateralen Kopf des M. gastrocnemius und des M. soleus unter stetiger Schonung der Muskelabgänge um den Fibulakopf. Zur übersichtlichen Darstellung der Frakturfragmente sowie zwecks Präparation des Plattenlagers kann eine proximale partielle Inzision des M. soleus am Fibulaköpfchen erfolgen. Eine Präparation der A. und V. poplitea kann stumpf erfolgen, ist aber nicht zwingend notwendig, da meist eine Schonung des Gefäß-Nerven-Bündels unter dem retrahierten Muskelbauch des lateralen Kopfes des M. gastrocnemius möglich ist. Die Präparation der Kniekehle bringt den M. popliteus zum Vorschein. Dieser ist durch seinen festen tendinösen Anteil ein entscheidender Stabilisator der posterolateralen Ecke und sollte daher unbedingt geschont werden. Der M. popliteus sowie der laterale Kopf des M. gastrocnemius werden mit einem Langenbeck Haken nach medial und kranial retrahiert, sodass Einsicht 

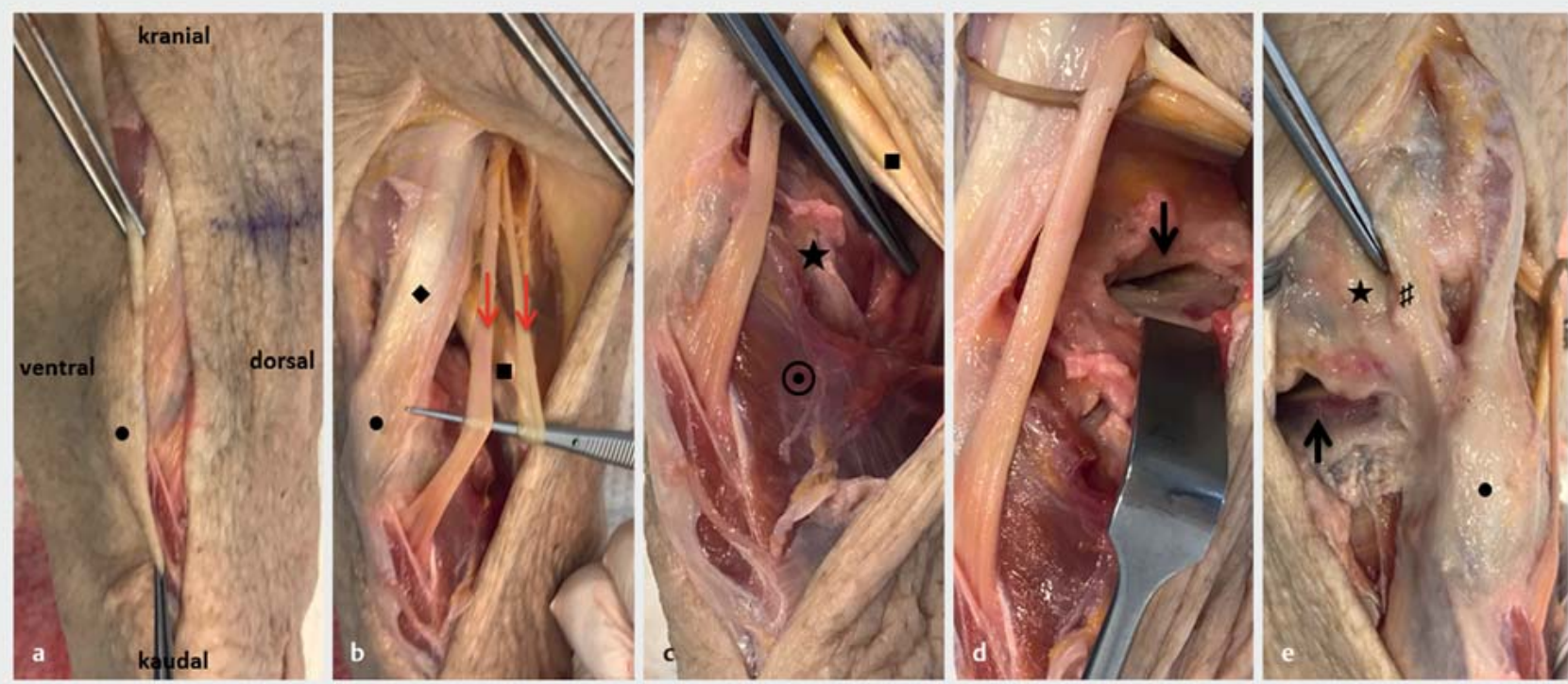

- Abb. 3 Posterolateraler Zugang nach Frosch in Seitenlage mit „Zwei-Fenster-Präparation“ dorsal und ventral des Fibulakopfes. a Hautschnitt beginnend etwa $4 \mathrm{~cm}$ oberhalb des Gelenkspaltes direkt über den Fibulakopf $(\bullet)$ verlaufend und nach distal ziehend. b Präparation des N. peronaeus $(\downarrow)$. In diesem Fall früher Abgang des N. cutaneus surae lateralis $(\downarrow \downarrow)$. Die Darstellung des N. peronaeus erfolgt entlang der Hinterkante des M. biceps femoris ( $\bullet$ über dem lateralen Gastroknemiuskopf ( • ). c Durch Retraktion des lateralen Gastroknemiuskopfes ( • ) nach medial kann das Gefäß-Nerven-Bündel potenziell dargestellt werden. Für gewöhnlich wird das Gefäß-Nerven-Bündel wie in diesem Fall unter dem retrahierten Muskelbauch geschont. d Anschließend vorsichtige Durchtrennung des proximalen Ansatzes des M. soleus ( $\odot)$ am Fibulaköpfchen, um nach entsprechender Arthrotomie über das posteriore Fenster (medial des Fibulakopfes) Einsicht auf die posterolaterale Gelenkfläche ( $\downarrow$ ) zu erlangen. e Ventral des Fibulakopfes $(\bullet)$ erfolgt die Präparation klassisch gemäß des anterolateralen Standardzugangs. Die Pinzette markiert hier das Intervall der tendinösen Anteile des M. popliteus $(\star)$ und des Außenbandes (\#). Die Arthrotomie $(\uparrow)$ erfolgt über das anteriore Fenster. Durch diese „Zwei-Fenster-Präparation“ kann visuelle Kontrolle über einen Großteil des lateralen Tibiaplateaus erlangt werden.

auf circa $20-30 \%$ des posterolateralen Tibiaplateaus geschaffen werden kann.

Ventral des Fibulakopfes wird die Präparation klassisch gemäß des anterolateralen Standardzugangs durchgeführt, sodass lateral das 2. Fenster entsteht. Die dargestellte „Zwei-Fenster-Präparation“ von dorsal und ventral des Fibulakopfes ermöglicht es, die visuelle Kontrolle über einen Großteil des lateralen Tibiaplateaus zu erlangen, um eine anatomische Reposition sowie die ideale osteosynthetische Stabilisierung mithilfe einer Abstützplatte oder Schrauben durchführen zu können [22] ( $\triangleright$ Abb. 3). Die zentralen Segmente des Tibiakopfes sind so jedoch nur schwer einsehbar.

\section{Merke}

Bei posterolateralen Frakturbeteiligungen sind ergänzende posterolaterale oder posteromediale Zugänge notwendig. Die Autoren halten den posterolateralen Zugang ohne Fibulaosteotomie mit Anlage eines posterioren und eines lateralen Fensters zur Visualisierung der Gelenkfläche für den geeignetsten Zugang.

\section{Erweiterter lateraler Zugang}

Bei umfassender Destruktion des lateralen Tibiaplateaus mit Beteiligung der zentralen Segmente kann ein erweiterter lateraler Zugang indiziert sein, um eine umfassende visuelle Gelenkeinsicht zu erlangen. Die Einsicht in die zentralen Segmente des Tibiakopfes über die Präparation des anterolateralen Zugangs (Darstellung des vorderen anterolateralen Drittels) und des posterolateralen Zugangs (Darstellung des nach dorsal abfallenden Gelenkabschnittes) ist erschwert und die ausschließliche intraoperative fluoroskopische Kontrolle mit dem Bildwandler gilt als Risikofaktor für das Persistieren von Gelenkstufen [26, 27].

Eine annähernd komplette Gelenkeinsicht kann mithilfe einer Osteotomie des Fibulakopfes oder der lateralen femoralen Epikondyle gelingen [28, 29]. Die Autoren bevorzugen die Durchführung einer Osteotomie der lateralen Femurepikondyle, da für den transfibulären Zugang mit Fibulakopfosteotomie sekundäre Repositionsverluste und Materialversagen nicht selten sind. Zudem besteht bei der Osteotomie der lateralen Femurepikondyle entgegen der Fibulakopfosteotomie keine erhöhte Verletzungsgefahr des N. peronaeus [30]. In den meisten Fällen ist eine Osteotomie des femoralen Ansatzes des lateralen 
Kollateralbands ausreichend, sodass die Sehne des M. popliteus von der Osteotomie unberührt bleibt und in ihrer stabilitätsgebenden Funktion nicht beeinflusst wird. Durch diese Form der Osteotomie kann einer Eröffnung des lateralen Gelenkspaltes um bis zu $7 \mathrm{~mm}$ erreicht werden, was einen guten Überblick auf die nahezu gesamte laterale Gelenkfläche ermöglicht ( $\bullet$ Abb. 4). Die Popliteussehne sollte nur im Falle sehr komplexer Frakturgeschehen in die Osteotomie mit einbezogen werden, falls auf anderem Wege keine ausreichende Visualisierung der beiden zentralen Tibiakopfsegmente erzielt werden kann. Für die Refixation der lateralen Femurepikondyle wird eine Zugschraubenosteosynthese mit 2 kanülierten (3,5-4 mm) Schrauben empfohlen.

\section{Cave}

Wichtig bei der Versorgung von Tibiakopffrakturen ist es, eine möglichst komplette visuelle Einsicht über die frakturierte Gelenkfläche zu erlangen, da die alleinige fluoroskopische Repositionskontrolle ein Risikofaktor für das Persistieren von intraartikulären Stufen ist!

\section{Anteromedialer Zugang}

Assoziiert mit bikondylären Verletzungen des Tibiakopfes finden sich mediale Frakturen des Tibiaplateaus häufig als posteromediale Abscherfrakturen [5]. Kommt es zum sehr seltenen Auftreten einer isolierten anteromedialen Impressions- oder Spaltfraktur, können diese über einen anteromedialen Zugang adressiert werden. Die Hautinzision sollte dabei so durchgeführt werden, dass selbiger Schnitt für den Fall einer eventuell später notwendigen Endoprothesenimplantation genutzt werden könnte. Über den anteromedialen Zugang gelingt die Visualisierung des vorderen Drittels der medialen Gelenkfläche. Hierfür wird die Gelenkkapsel in Längsrichtung inzidiert und die Arthrotomie nach horizontalem Absetzen meniskotibialer Fasern im Bereich ihres tibialen Ansatzes durchgeführt. Die weitere Einsicht nach dorsal auf die mediale Gelenkfläche bleibt dem Operateur durch die oberflächlichen Anteile des medialen Kollateralbandes, welches nicht durchtrennt werden sollte, verwehrt. Für den Fall, dass auch die zentral gelegenen Areale der medialen Gelenkfläche dargestellt werden müssen, kann eine Erweiterung des Zugangs durch eine Osteotomie des medialen Femurepikondylus erfolgen. Der anteromediale Zugang eignet sich vor allem in Kombination mit dem anterolateralen oder dem lateralen Zugang, wobei die Breite der Weichteilbrücke mindestens $7 \mathrm{~cm}$ betragen sollte, um die Entstehung von Nekrosen oder Wundheilungsstörungen zu vermeiden [31].

\section{Merke}

Falls die Visualisierung zentral gelegener Areale der medialen Gelenkfläche notwendig ist, kann eine Erweiterung des anteromedialen Zugangs mittels Osteotomie des medialen Femurepikondylus erfolgen.

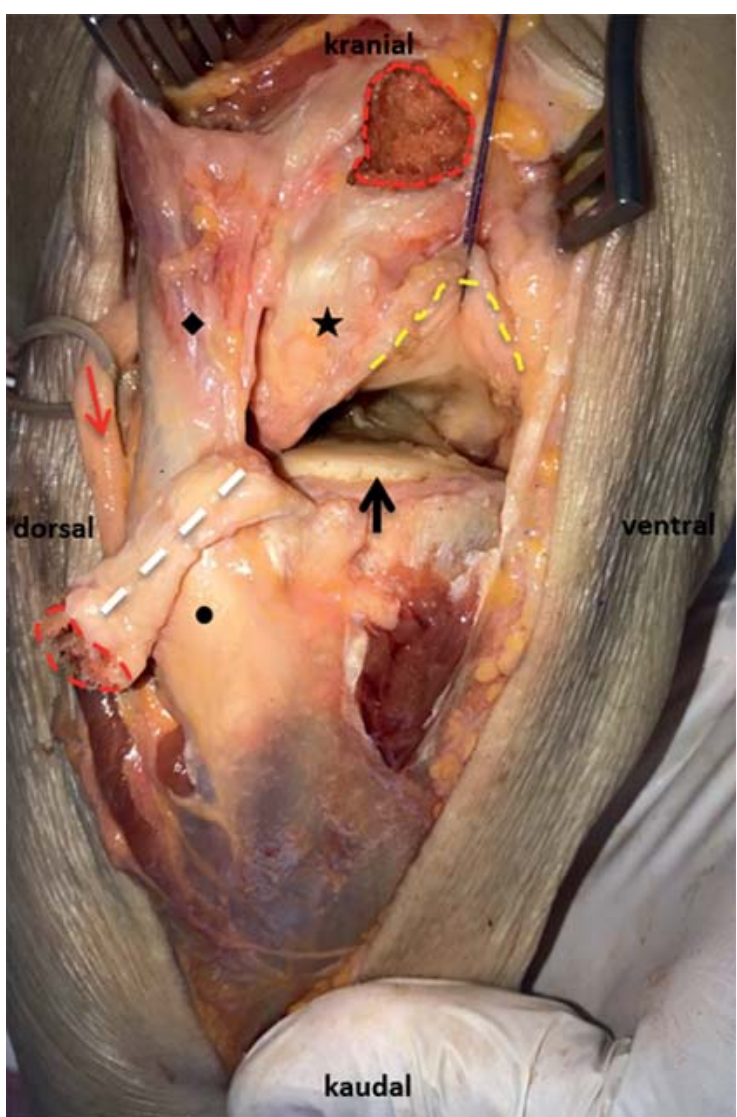

- Abb. 4 Osteotomie der lateralen Femurepikondyle im Rahmen des posterolateralen Zugangs in Seitenlage zur erweiterten Visualisierung der lateralen Gelenkfläche über das anteriore Fenster. Bereits erfolgte Osteotomie eines etwa 1,5 × 1,5 ×0,5 cm messenden Knochenblocks (grob-gestrichelter roter Kreis) mit lateralem Kollateralband (LCL, gestrichelte weiße Linie), welches sich zuvor ventral des M. biceps femoris aufspannte $(\bullet)$. Dorsal davon angeschlungen der $N$. peronaeus $(\downarrow)$. Ventral des M. biceps femoris ist der M. popliteus $(\star)$ dargestellt. Ersichtliche Osteotomiestelle an der lateralen Femurepikondyle (fein-gestrichelter roter Kreis). Deutlich verbesserte Gelenkeinsicht $(\uparrow$ ) submeniskal durch kraniale Retraktion des angeschlungenen Außenmeniskus (gestrichelte gelbe Linie).

\section{Posteromediale Zugänge in Rücken- oder Bauchlage}

Durch den posteromedialen Zugang nach Lobenhoffer gelingt eine gute Visualisierung im Bereich der dorsome-

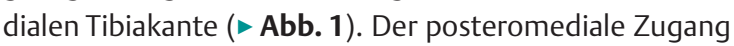
kann hilfreich bei der Versorgung der besonders häufigen bikondylären Frakturen mit mehrheitlich medialer, koronarer Splitfraktur sein. Die Rekonstruktion der medialen Säule genießt dabei Priorität und sollte in der Regel vor Adressierung des lateralen Tibiaplateaus erfolgen, da das mediale Tibiaplateau als Referenz für die korrekte Achse, Länge und Breite des Tibiakopfes dienen kann. Auch die distalen Frakturausläufer der häufig mit bikondylären Verletzungen einhergehenden posteromedialen Abscherungen des Tibiaplateaus können über den posterome- 

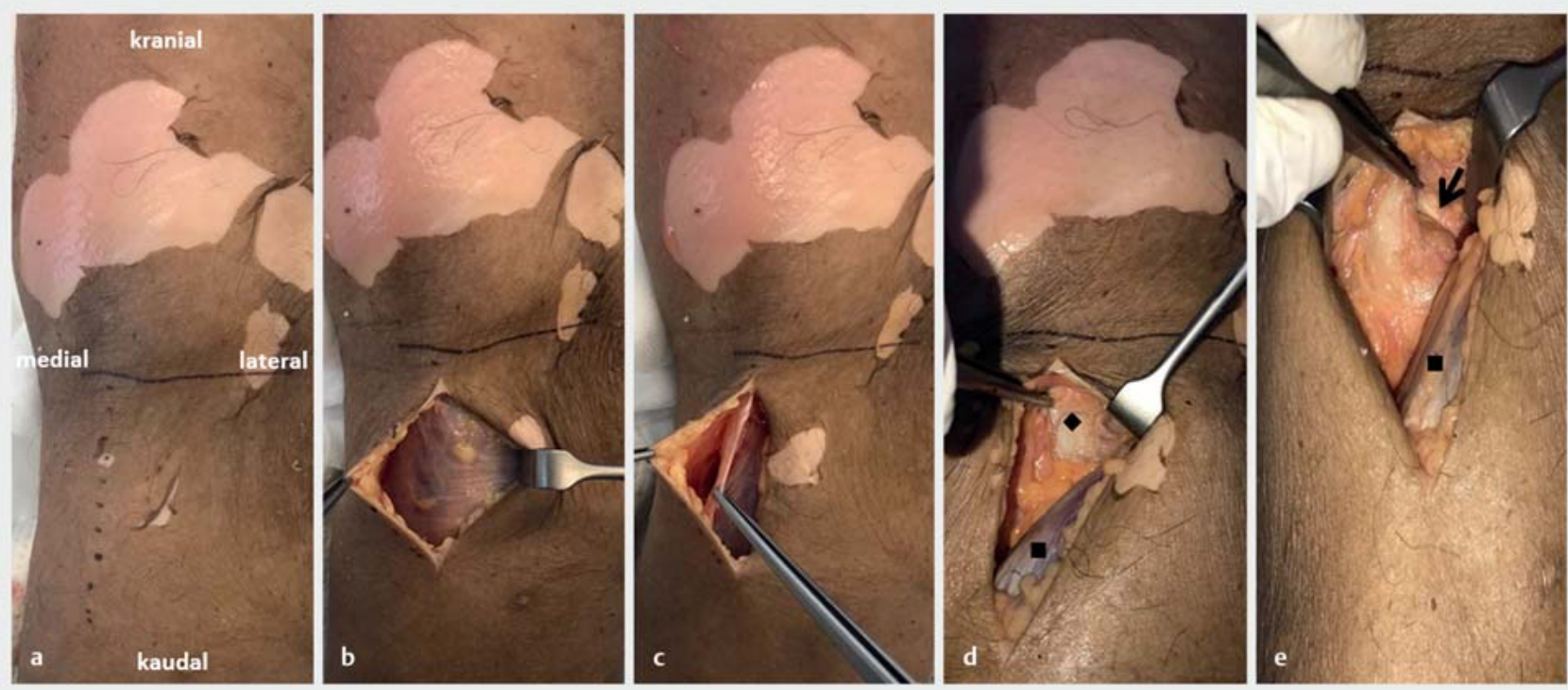

- Abb. 5 Posteromedialer Zugang in Bauchlage. a, b Der Hautschnitt erfolgt in Bauchlage über dem medialen Kopf des M. gastrocnemius. Nach Eröffnung der Haut und der Subkutis fällt der Blick auf die Fascia cruris. c Die Fascia cruris wird mit der Pinzette nach medial mobilisiert. $\mathbf{d}$ Unter stumpfem digitalen Austasten und Retraktion des medialen Kopfes des M. gastrocnemius ( • ) nach lateral erfolgt die Freilegung des M. semimembranosus $(\bullet)$. e Dieser wird nach medial retrahiert (Pinzette), sodass ein Blick auf die dorsomedialen Anteile der Gelenkkapsel möglich ist und eine posteromediale Arthrotomie durchgeführt werden kann $(\downarrow)$.

dialen Zugang gut dargestellt und adressiert werden [29].

Der Hautschnitt wird bei dem auf dem Rücken mit leicht gebeugtem Knie oder in Vierer-Position gelagerten Patienten über der dorsomedialen Tibiakante frakturabhängig über eine ungefähre Länge von $10 \mathrm{~cm}$ durchgeführt. Es folgt die Präparation der Sehnen der „Hamstrings“, der Zugang zum posteromedialen Tibiakopf erfolgt dann entweder zwischen den Sehnen hindurch oder unter Retraktion an diesen vorbei. Mithilfe einer posteromedialen Abstützplatte gelingt in den meisten Fällen durch die Reposition extraartikulärer distaler Frakturausläufer eine anatomisch, stufenlose Wiederherstellung der Gelenkfläche. Im Falle einer dorsalen Trümmerzone mit mehrfragmentärer Gelenkbeteiligung oder einem größeren Gelenkblock mit posterolateralzentralen Anteilen (häufig bei Moore-II-Frakturen) gelingt in Rückenlage oft keine ausreichende Darstellung, sodass ein direkter posteromedialer Zugang in Bauchlage durchgeführt werden sollte. Der längsverlaufende Hautschnitt erfolgt in Bauchlage über dem medialen Kopf des M. gastrocnemius ( $\triangleright$ Abb. 5). Zu beachten ist dabei, dass die Inzision die Beugefalte der Kniekehle nicht kreuzen sollte, um Bewegungseinschränkungen durch Narbenkontrakturen zu vermeiden. Anschließend erfolgt die Präparation bis auf die Faszie des M. gastrocnemius, durch stumpfe digitoklastische Mobilisation zwischen dem medialen Kopf des M. gastrocnemius und dem M. semimembranosus. Die Darstellung der dorsomedialen Anteile der Gelenkkapsel gelingt durch Retraktion des Gastroknemiuskopfes nach lateral unter Schonung des Gefäß-Nerven-Bündels ( $\vee$ Abb. 5). Aufgrund einer Adipositas oder bei Patienten mit kräftiger Muskulatur muss eine Erweiterung des Hautschnittes entlang der Beugefalte nach lateral in Erwägung gezogen werden, wodurch die Visualisierung in das posterolateralzentrale Tibiaplateau verbessert werden kann. Zudem kann eine partielle Inzision des tendinösen Ansatzes des M. gastrocnemius femoral erfolgen, um die Darstellung der Fraktur zu verbessern. Bei der Durchführung der posteromedialen Arthrotomie sollte in jedem Fall der sich lateral befindende Ansatz des hinteren Kreuzbands und die medial gelegenen Ansätze des M. semimembranosus geschont und nicht verletzt werden. Eine partielle proximale Lösung des M. soleus und des M. popliteus zur Präparation des posteromedialen Plattenlagers ist möglich.

\section{Cave}

Im Rahmen der Arthrotomie über den posteromedialen Zugang ist Vorsicht geboten, um den Ansatz des hinteren Kreuzbandes und des M. semimembranosus nicht akzidentell zu verletzen!

\section{Erweiterter medialer Zugang}

Mehrfragmentäre mediale Trümmerzonen sind durch die breite Auflage des medialen Kollateralbandes, den Verlauf des medialen hinteren Schrägbandes (POL) sowie der Konkavität des medialen Tibiaplateaus nicht suffizient über einen antero- oder posteromedialen Zugang dar- 


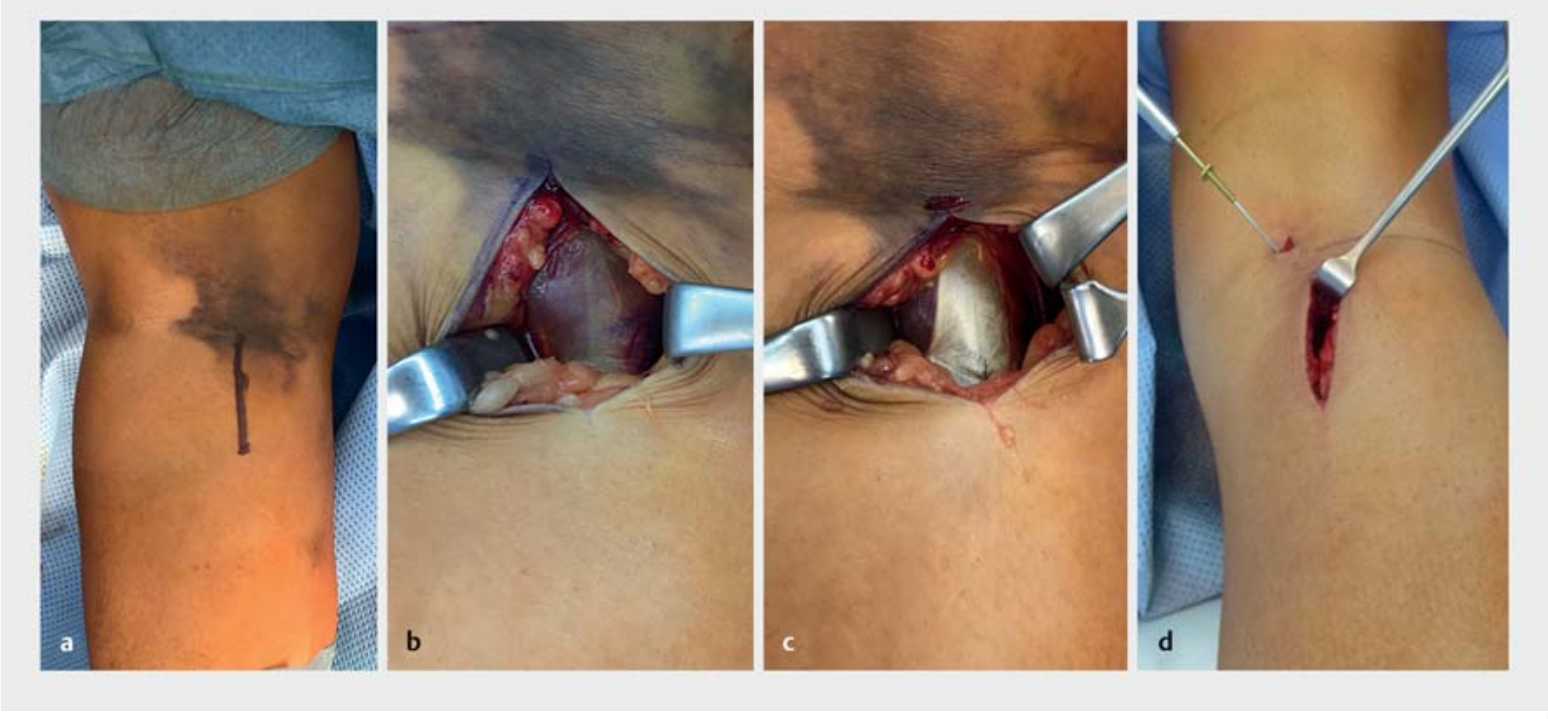

Abb. 6 Minimalinvasive Versorgung eines knöchernen hinteren Kreuzbandausrisses [23]. Intraoperative Fotodokumentation. a Bauchlagerung des Patienten. b Circa 3-4 cm lange Hautinzision direkt über dem Muskelbauch des medialen Kopfes des M. gastrocnemius. Nach Durchtrennung des subkutanen Fettgewebes kommt zunächst die Fascia cruris zum Vorschein. c Nach Durchtrennung der Fascia cruris wird der mediale Gastroknemiuskopf nach lateral retrahiert. Das Gefäß-Nerven-Bündel kommt geschützt unterhalb des medialen Gastroknemiuskopfes zu liegen und muss nicht dargestellt werden. $\mathbf{d}$ Nach erfolgter Frakturreposition erfolgt die Fixation mit 2 Bohrdrähten. Diese kann, wie hier in einem anderen Fall dargestellt, wahlweise über ein

2. minimalinvasives Fenster von medial durchgeführt werden (Cave: Gefäße und Nerven liegen mittig oder posterolateral!). Nach Überbohrung folgt das Einbringen von 2 kanülierten Titanschrauben mit Unterlegscheibe zur „wasserdichten“ Fixierung des reponierten Ausrissfragmentes.

stellbar. Daher kann die Durchführung einer weichteilschonenden Osteotomie des medialen femoralen Epikondylus zu einer verbesserten Einsichtnahme notwendig werden. Analog zum erweiterten lateralen Zugang erfolgt dabei medial die Osteotomie eines circa $2 \times 2 \times 1 \mathrm{~cm}$ großen Knochenblocks, der anschließend mit 2 Spongiosaschrauben refixiert werden kann. Durch die Osteotomie gelingt eine erweiterte Einsicht auf ca. 70\% des medialen Tibiaplateaus - das dorsale Drittel kann über den erweiterten medialen Zugang alleine nicht eingesehen werden.

\section{Minimalinvasive Zugänge}

In dieser Übersicht der chirurgischen Zugänge zum Tibiakopf werden die minimalinvasiven Zugänge zwar zuletzt aufgeführt, im klinischen Alltag sind sie aber von Wichtigkeit, um eine umfassende, weichteilschonende und erfolgsorientierte Versorgung von Tibiakopffrakturen gewährleisten zu können.

Wenig dislozierte Frakturen, für die eine operative Versorgungsindikation besteht, können in minimalinvasiver Technik versorgt werden. Mithilfe einer kortikalen Fensterung kann eine Impressionsfraktur unter Zuhilfenahme eines Stößels unter arthroskopischer Kontrolle aufgerichtet werden und anschließend über einen anterolateralen Zugang mittels Plattenosteosynthese oder über senkrecht zueinander eingebrachte kanülierte 3,5-mm-
Schrauben stabilisiert werden. In biomechanischen Studien zeigte sich eine verbesserte Verteilung der Last mit einem geringeren Risiko des sekundären Schrauben-Auswanderns, wenn bei dieser sogenannten „Jail-Technik“ die Jail-Schrauben genau unterhalb der fixierenden Schrauben eingebracht wurden [32].

Die konventionelle Arthroskopie, die normalerweise mit einem erhöhten intraartikulären Wasserdruck einhergeht, eignet sich bei AO-Typ-B-Frakturen ohne einhergehende Verletzung der Kapsel, da andernfalls (bei TypC-Frakturen) ein iatrogenes Kompartmentsyndrom entstehen kann. Bei dem Einsatz einer arthroskopischen Optik ohne erhöhten Spüldruck, eingebracht über den chirurgischen Zugang, spricht man von der sogenannten „Frakturoskopie“. Die Frakturoskopie ist inzwischen auch bei komplexen Tibiakopffrakturen zur verbesserten intraoperativen Visualisierung der Gelenkfläche ein etabliertes Verfahren [26,33].

Die Versorgung knöcherner Ausrisse des hinteren Kreuzbandes gelingt über einen minimalinvasiven dorsalen Zugang [23] (• Abb. 6 und 7). Die Lagerung des Patienten erfolgt hierfür in Bauchlage, die etwa $3-4 \mathrm{~cm}$ lange Hautinzision erfolgt direkt über dem Muskelbauch des medialen Kopfes des M. gastrocnemius. Nach anschließender Durchtrennung der Subkutis erfolgt die Eröffnung der Faszie und der Muskelbauch des medialen Gastroknemi- 


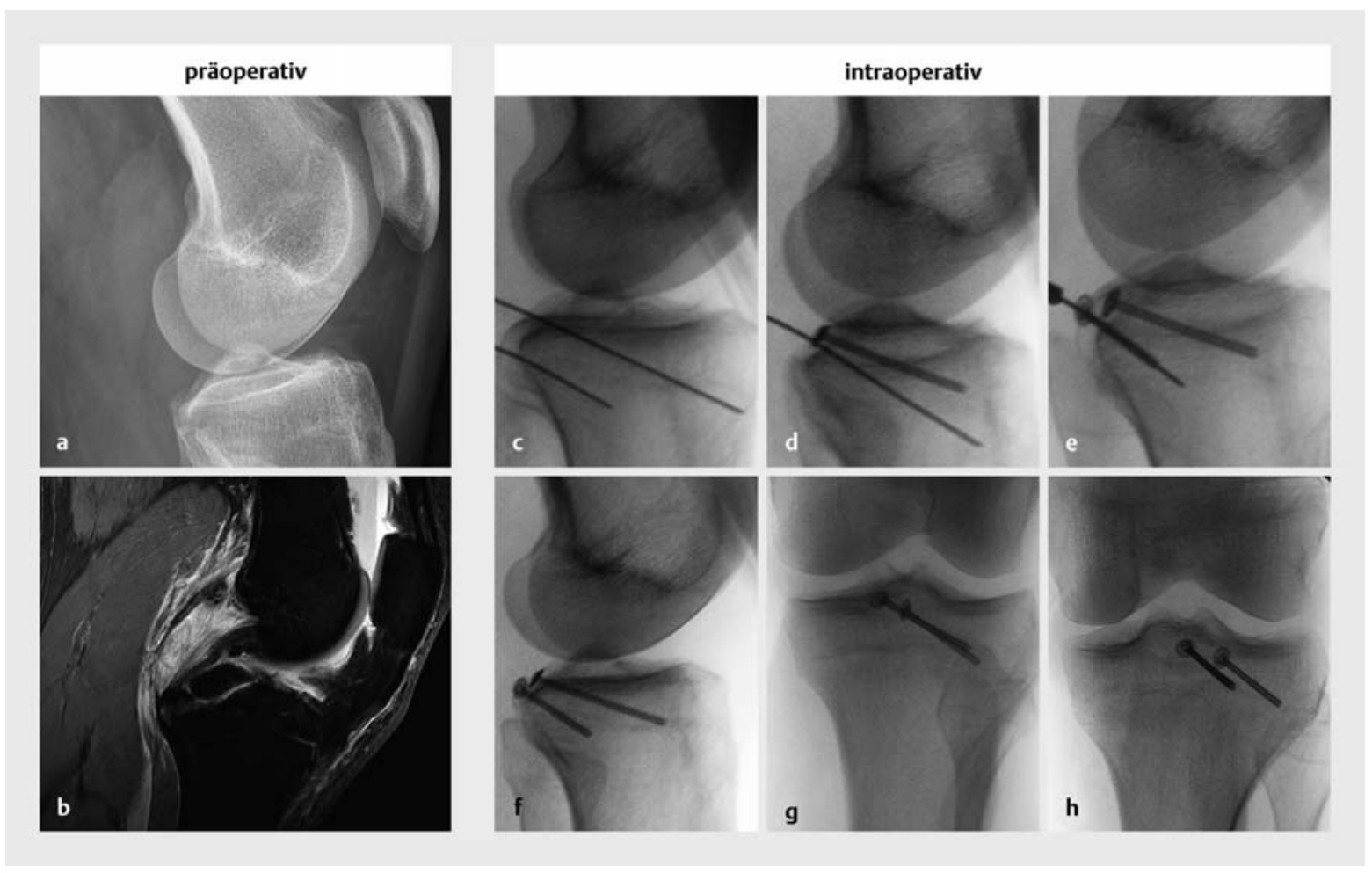

- Abb. 7 Prä- und intraoperative Bildgebung. a In der präoperativen Röntgendiagnostik lässt sich die Verletzung bereits erkennen und $\mathbf{b}$ im MRT bestätigen. c-e Durch die intraoperative Fluoroskopie kann eine kontinuierliche Lage- und Repositionskontrolle erfolgen. f-h Das knöcherne Ausrissfragment zeigt sich anatomisch reponiert und stabil mit 2 kanülierten Titanschrauben fixiert.

uskopfes wird nach lateral retrahiert ( $\bullet$ Abb. 6). Das Gefäß-Nerven-Bündel liegt geschützt unter dem medialen Gastroknemiuskopf und wird dementsprechend mit nach lateral weggehalten; es sollte nicht dargestellt werden. Eine Inzision am tendinösen proximalen Ansatz des medialen Gastroknemiuskopfes zum „Release“ zwecks Verbesserung der Übersicht ist möglich. Nach Austasten der interkondylären Grube am dorsalen Tibiakopf kann die dorsale Kapsel scharf vom Tibiakopf von distal nach proximal unter Schonung des hinteren Kreuzbandes abgelöst werden. Nach entsprechender Frakturreposition erfolgt die Fixation mit 2 Bohrdrähten und deren Überbohrung, um mit 2 kanülierten Titanschrauben das reponierte Frakturfragment zu fixieren ( $\triangleright$ Abb. 7).

\section{Merke}

- Wenig dislozierte Frakturen des Tibiakopfes können unter arthroskopisch gestützter Kontrolle mittels Schraubenosteosynthese in „Jail“-Technik versorgt werden.

- Die sogenannte „Frakturoskopie“ kann bei komplexen Frakturmustern zur intraoperativen Darstellung der Gelenkfläche hilfreich sein.

- Die Versorgung von knöchernen Ausrissen des hinteren Kreuzbandes kann über einen minimalinvasiven dorsomedialen Zugang erfolgen, der technisch einfach und komplikationsarm ist.

\section{Fazit}

- Die Klassifikation von Tibiakopffrakturen sollte behandlungsorientiert sein und die Fraktur in ihrer Dreidimensionalität erfasst werden. Die Autoren präferieren die AO- sowie die Zehensegmentklassifikation.

- Die Morphologie der Fraktur gibt den idealen chirurgischen Zugang oder die Kombination der Zugänge vor.

- Von besonderer Wichtigkeit ist es, eine möglichst komplette visuelle Einsicht über die frakturierten Gelenkflächen zu erlangen. Die alleinige fluoroskopische Repositionskontrolle reicht meist nicht aus und gilt als Risikofaktor für das Persistieren von intraartikulären Stufen.

- Zum Erlangen einer ausreichenden Übersicht können die verschiedenen Zugänge kombiniert oder ggf. erweitert werden. Dabei kann durch eine laterale oder mediale femorale Epikondylenosteotomie jeweils die Einsicht auf die betroffene Gelenkfläche entscheidend verbessert werden.

- Die Autoren halten den posterolateralen Zugang nach Frosch für einen geeigneten Zugang, um eine gute Übersicht über die postero- und anterolateralen Quadranten des Tibiakopfes zu erlangen. Eine Osteotomie des Fibulakopfes ist in der Regel nicht notwendig.

- Minimalinvasive Eingriffe spielen bei der Versorgung der Tibiakopffraktur ebenfalls eine große Rolle. Ar- 
throskopisch gestützte Osteosyntheseverfahren, die „Frakturoskopie“ sowie der direkte dorsale Zugang zur Versorgung von knöchernen Ausrissen des hinteren Kreuzbandes sind etablierte und hilfreiche Verfahren.

\section{Interessenkonflikt}

Herr Professor Frosch hat Referentenvereinbarungen mit der AE, Arthrex und Rimasys

\section{Autorinnen/Autoren}

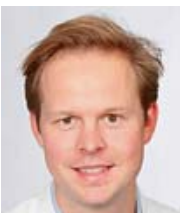

\section{Tim-Nicolas Hissnauer}

Dr. med., wissenschaftlicher Mitarbeiter, Klinik und Poliklinik für Unfall-, Hand- und Wiederherstellungschirurgie, Universitätsklinikum Hamburg-Eppendorf

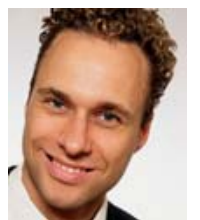

\section{Matthias Krause}

PD Dr. med., wissenschaftlicher Mitarbeiter Klinik und Poliklinik für Unfall-, Hand- und Wiederherstellungschirurgie, Universitätsklinikum Hamburg-Eppendorf

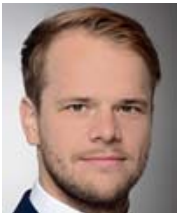

\section{Jannik Frings}

Dr. med., wissenschaftlicher Mitarbeiter, Klinik und Poliklinik für Unfall-, Hand- und Wiederherstellungschirurgie, Universitätsklinikum Hamburg-Eppendorf

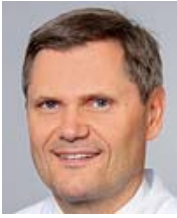

\section{Karl-Heinz Frosch}

Univ.-Prof. Dr. med., Klinikdirektor, Klinik und Poliklinik für Unfall-, Hand- und Wiederherstellungschirurgie, Universitätsklinikum HamburgEppendorf

\section{Korrespondenzadresse}

Univ.-Prof. Dr. med. Karl-Heinz Frosch

Klinik und Poliklinik für Unfall-, Hand- und

Wiederherstellungschirurgie

Universitätsklinikum Hamburg-Eppendorf

Martinistraße 52

20246 Hamburg

Tel.: 0 40/74 10-53459

Fax: 040/7410-54569

k.frosch@uke.de

\section{Literatur}

[1] Elsoe R, Larsen P, Nielsen NP et al. Population-Based Epidemiology of Tibial Plateau Fractures. Orthopedics 2015; 38: e780e786. doi:10.3928/01477447-20150902-55

[2] Papagelopoulos PJ, Partsinevelos AA, Themistocleous GS et al. Complications after tibia plateau fracture surgery. Injury 2006; 37: 475-484. doi:10.1016/j.injury.2005.06.035

[3] Rossbach BP, Faymonville C, Muller LP et al. [Quality of life and job performance resulting from operatively treated tibial pla- teau fractures]. Unfallchirurg 2016; 119: 27-35. doi:10.1007| s00113-014-2618-z

[4] Manidakis N, Dosani A, Dimitriou R et al. Tibial plateau fractures: functional outcome and incidence of osteoarthritis in 125 cases. Int Orthop 2010; 34: 565-570. doi:10.1007| s00264-009-0790-5

[5] Krause M, Preiss A, Muller G et al. Intra-articular tibial plateau fracture characteristics according to the "Ten segment classification”. Injury 2016; 47: 2551-2557. doi:10.1016/j.injury.2016.09.014

[6] Honkonen SE. Degenerative arthritis after tibial plateau fractures. J Orthop Trauma 1995; 9: 273-277

[7] Enderle E, Frosch KH. [Arthroscopy-assisted management of knee fractures]. Unfallchirurg 2013; 116: 311-317. doi:10.1007/s00113-012-2346-1

[8] Doornberg JN, Rademakers MV, van den Bekerom MP et al. Two-dimensional and three-dimensional computed tomography for the classification and characterisation of tibial plateau fractures. Injury 2011; 42: 1416-1425. doi:10.1016/j.injury.2011.03.025

[9] Marsh JL, Slongo TF, Agel ] et al. Fracture and dislocation classification compendium - 2007: Orthopaedic Trauma Association classification, database and outcomes committee. J Orthop Trauma 2007; 21: S1-S133

[10] Schatzker J MR, Bruce D. The tibial plateau fracture. The Toronto experience 1968-1975. Clin Orthop Relat Res 1979; (138): 94-104

[11] Luo CF, Sun H, Zhang B et al. Three-column fixation for complex tibial plateau fractures. J Orthop Trauma 2010; 24: 683692. doi:10.1097/BOT.0b013e3181d436f3

[12] Wang Y, Luo C, Zhu Y et al. Updated Three-Column Concept in surgical treatment for tibial plateau fractures - A prospective cohort study of 287 patients. Injury 2016; 47: 1488-1496. doi:10.1016/j.injury.2016.04.026

[13] Krause M, Frosch KH. Response to the letter-to-the-editor by Dhillon et al. "Simple four column classification can dictate treatment for intra articular tibial plateau fractures much better than ten segment classification", Injury 2017. Injury 2017; 48: 2369-2370. doi:10.1016/j.injury.2017.07.040

[14] Raschke M], Kittl C, Domnick C. Partial proximal tibia fractures. EFORT Open Rev 2017; 2: 241-249. doi:10.1302/20585241.2.160067

[15] Mthethwa J, Chikate A. A review of the management of tibial plateau fractures. Musculoskelet Surg 2018; 102: 119-127. doi:10.1007/s12306-017-0514-8

[16] Xu YQ, Li Q, Shen TG et al. An efficacy analysis of surgical timing and procedures for high-energy complex tibial plateau fractures. Orthop Surg 2013; 5: 188-195. doi:10.1111/ os. 12057

[17] Ruffolo MR, Gettys FK, Montijo HE et al. Complications of high-energy bicondylar tibial plateau fractures treated with dual plating through 2 incisions. J Orthop Trauma 2015; 29: 85-90. doi:10.1097/BOT.0000000000000203

[18] Dubina AG, Paryavi E, Manson TT et al. Surgical site infection in tibial plateau fractures with ipsilateral compartment syndrome. Injury 2017; 48: 495-500. doi:10.1016/j.injury.2016.10.017

[19] Zura RD, Adams SB jr., Jeray KJ et al. Timing of definitive fixation of severe tibial plateau fractures with compartment syndrome does not have an effect on the rate of infection. J Trauma 2010; 69: 1523-1526. doi:10.1097/ TA.0b013e3181d40403 
[20] Krause M, Menzdorf L, Preiss A et al. Are there four tibial plateau columns? Yes there are, as illustrated by a postero-lateral apple-bite fracture. Response to a letter-to-the-editor. Int Orthop 2018; 42: 443-446. doi:10.1007/s00264-017-3686-9

[21] Solomon LB, Stevenson AW, Lee YC et al. Posterolateral and anterolateral approaches to unicondylar posterolateral tibial plateau fractures: a comparative study. Injury 2013; 44: 1561-1568. doi:10.1016/j.injury.2013.04.024

[22] Frosch KH, Balcarek P, Walde T et al. A new posterolateral approach without fibula osteotomy for the treatment of tibial plateau fractures. J Orthop Trauma 2010; 24: 515-520. doi:10.1097/BOT.0b013e3181e5e17d

[23] Frosch K, Proksch N, Preiss A et al. [Treatment of bony avulsions of the posterior cruciate ligament (PCL) by a minimally invasive dorsal approach]. Oper Orthop Traumatol 2012; 24: 348-353. doi:10.1007/s00064-012-0208-1

[24] Hoekstra H. Are there four tibia plateau columns? Int Orthop 2017; 41: 2631-2632. doi:10.1007/s00264-017-3602-3

[25] Cho JW, Kim J, Cho WT et al. Approaches and fixation of the posterolateral fracture fragment in tibial plateau fractures: a review with an emphasis on rim plating via modified anterolateral approach. Int Orthop 2017; 41: 1887-1897. doi:10.1007| s00264-017-3563-6

[26] Krause M, Preiss A, Meenen NM et al. "Fracturoscopy" is Superior to Fluoroscopy in the Articular Reconstruction of Complex Tibial Plateau Fractures-An Arthroscopy Assisted Fracture Reduction Technique. J Orthop Trauma 2016; 30: 437-444. doi:10.1097/BOT.0000000000000569

[27] Reul M, Nijs S, Rommens PM et al. [Intra-articulair tibial Plateau Fractures]. Z Orthop Unfall 2017; 155: 352-370. doi:10.1055/s-0042-116779
[28] Bowers AL, Huffman GR. Lateral femoral epicondylar osteotomy: an extensile posterolateral knee approach. Clin Orthop Relat Res 2008; 466: 1671-1677. doi:10.1007/s11999-0080232-5

[29] Lobenhoffer P, Gerich T, Bertram T et al. [Particular posteromedial and posterolateral approaches for the treatment of tibial head fractures]. Unfallchirurg 1997; 100: 957-967

[30] Pires RES, Giordano V, Wajnsztejn A et al. Complications and outcomes of the transfibular approach for posterolateral fractures of the tibial plateau. Injury 2016; 47: 2320-2325. doi:10.1016/j.injury.2016.07.010

[31] Krause M, Muller G, Frosch KH. [Surgical approaches to tibial plateau fractures]. Unfallchirurg 2018. doi:10.1007/s00113018-0515-6

[32] Weimann A, Heinkele T, Herbort M et al. Minimally invasive reconstruction of lateral tibial plateau fractures using the jail technique: a biomechanical study. BMC Musculoskelet Disord 2013; 14: 120. doi:10.1186/1471-2474-14-120

[33] Krause M, Preiss A, Frosch KH. Fracturoscopy. Arthroskopie 2018; 31: 59-65. doi:10.1007/s00142-017-0181-7

\section{Bibliografie}

DOI https://doi.org/10.1055/a-0785-1655

Online-publiziert 04.02.2019 | OP-JOURNAL 2019; 35: 107116 ๑ Georg Thieme Verlag KG Stuttgart · New York ISSN 0178-1715 